\title{
Beitrag zur \\ Kenntnis der Blaualgenvegetation der Thermen von Baden und Leukerbad (Schweiz)
}

(Vorläufige Mitteilung)

Von Konstantin ANAGNOSTIDIS und ALFONS ZEHNDER

(Aus dem Institut für systematische Botanik und Pflanzengeographie der Universität Thessaloniki und der Eidgenössischen Anstalt für Wasserversorgung, Abwasserreinigung und Gewässerschutz an der ETH, Zürich.) 


\title{
Beitrag zur \\ Kenntnis der Blaualgenvegetation der Thermen von Baden und Leukerbad (Schweiz)
}

\author{
(Vorläufige Mitteilung) \\ Von Konstantin ANagnostidis und ALFons ZeHnder \\ (Aus dem Institut für systematische Botanik und Pflanzengeographic \\ der Universität Thessaloniki und der Eidgenössischen Anstalt für Wasserversorgung, \\ Abwasserreinigung und Gewässerschutz an der ETH, Zürich.)
}

Manuskript eingegangen am 2I. April r964

\begin{abstract}
A. Einleitung
Im Spätsommer 1963 fand im Hydrobiologischen Laboratorium der ETH in Kastanienbaum am Vierwaldstättersee (Schweiz) das dritte Symposium der Internationalen Arbeitsgemeinschaft für Cyanophytenkunde (IAC) statt [8]. Exkursionen führten die Teilnehmer unter anderem zu den Thermen von Baden (Aargau) und Leukerbad (Wallis), deren Algenvegetation bisher nie untersucht worden war. Die gesammelten Materialien überraschten durch ihren Artenreichtum; gleichzeitig tauchten bei der Analyse aber auch viele kritische Fragen auf, deren Klärung reichlich Zeit und eingehendere Untersuchungen an Ort und Stelle erfordern wird. So entschlossen wir uns, in dieser ersten Mitteilung eine vorläufige Übersicht über die festgestellten Taxa zu geben. Die Listen erheben keinen Anspruch auf Vollständigkeit; nicht eindeutig bestimmbare kritische Formen werden meist nicht erwähnt; ebenso verzichten wir auf die Angabe von « Varietäten», «Formen» usw. Für die Bestimmungen wurden hauptsächlich die Werke von DesiKachary [9], ELENKIN [10] und GEITLER [Ir] benützt. Wir hoffen, später eine ausführlichere Arbeit über die Algenvegetation der beiden Thermen vorlegen zu können.
\end{abstract}




\section{B. Baden}

Die I8 gefassten Thermalquellen von Baden und Ennetbaden [2-5] fördern täglich etwa I Million 1 Wasser aus der Muschelkalkformation durch Spalten im darüber lagernden Keuper an die Oberfläche. Sie lassen sich als Kochsalz- und Gipsthermen mit mildem Schwefelwasserstoffgehalt charakterisieren. Einige Angaben über Chemismus und physikalische Eigenschaften sind in Tab. I zusammengestellt; im übrigen sei auf die ausfïhrliche Monographie MüNZELs [s] verwiesen.

\section{Tabelle 1}

Das Thermalwasser der Verenaquelle pon Baden (Schweiz) (gekürzt nach GüBELI und DORTA-SCHAEPPI [2])

\begin{tabular}{|c|c|c|c|}
\hline \multicolumn{4}{|c|}{ Mineralgehalt (mg pro Liter) } \\
\hline \multicolumn{2}{|l|}{ Kationen } & \multicolumn{2}{|l|}{ Anionen } \\
\hline Natrium & $7 \mathbf{I} 2,00$ & Chlorid & II 18,49 \\
\hline Kalium & 59,45 & Sulfat & I365,0 \\
\hline Magnesium & 100,8 & Hydrokarbonat & 457,34 \\
\hline Kalzium & 523,88 & & \\
\hline Ubrige Kationen & II, $78 \mathrm{I}$ & Übrige Anionen & $8, \mathbf{I} 96$ \\
\hline Summe der Kationen & $\mathbf{I} 407,9 \mathrm{rr}$ & Summe der Anionen & 2949,026 \\
\hline Borsäure & 3,429 & & s \\
\hline Kieselsäure & 56,59 & · & \\
\hline Total Mineralien & $44 \mathrm{I} 6,956$ & & \\
\hline \multicolumn{4}{|c|}{ Gase: Kohlendioxyd, Schwefelwasserstoff } \\
\hline \multicolumn{4}{|c|}{ Temperatur: $46,09^{\circ} \mathrm{C}$ (hyperthermal) } \\
\hline
\end{tabular}

Von den Quellen wird das Wasser in unterirdischen Leitungen grösstenteils in die Badehotels geleitet. Als Algenstandorte kommen daher praktisch nur die wenigen im Freien liegenden Thermaltrinkbrunnen sowie die Bäderabläufe, die an der Ufermauer der Limmat ausmünden, in Betracht.

\section{Algen im Trinkbrumen der Kuranlage}

Der offene Trinkbrunnen in der Kuranlage der «Grossen Bäder», der von der benachbarten «Limmatquelle» aus gespeist wird, zeigte an seinem inneren Trogrand lockere, blaugrüne Algenbäuschchen von etwa $\mathrm{I} \mathrm{cm}$ Durchmesser. Die Wassertemperatur an den Algenstandorten betrug $39,5^{\circ} \mathrm{C}$. Die Analyse ergab:

plectonema notatum Schmidle

Filamente $2-2,4 \mu$ breit, Zellen $\mathrm{I}-\mathrm{I}, 5 \mu$ breit, 3-4,5 $\mu$ lang. Scheiden fest, farblos;

Scheinverzweigungen spärlich. 
Phormidium angustissimum W. et G. S. West

Ph. bigranulatum Gardner (?)

Filamente etwa i $\mu$ breit, sehr lang, ziemlich parallel gelagert und gerade oder regellos gelagert und mässig gebogen. Scheiden dünn, meist fast unsichtbar. Zellen $0,6-0,8 \mu$ breit, 2-8 $\mu$ lang, an den Querwänden nicht eingeschnürt; diese deutlich, oft mit je einem Körnchen auf jeder Seite.

Oscillatoria terebriformis Ag. ex Gom.

o.sp.

Zellen $0,8-1,2 \mu$ breit, bis smal länger als breit, blassblaugrün, an den Querwänden leicht eingeschnürt, mit je I Körnchen. Trichomenden gerade, nicht verjüngt, Endzelle abgerundet.

Beggiatoa minima Winogr.

B. leptomitiformis (Menegh.) Trevis

Tbiotbrix tenuis Winogr.

Diatomeen

\section{Algen im Auslauf des Kurbrunnenwassers in die Limnat}

Das Überlaufwasser des Kurbrunnens wird unterirdisch weggeführt und tritt etwa $50 \mathrm{~cm}$ über dem mittleren Wasserspiegel der Limmat aus der Ufermauer aus. Seine Temperatur misst hier noch $35,5^{\circ} \mathrm{C}$. Unterhalb der Ausmündung hat sich eine dicke Tuffkruste angesetzt, über die das Wasser herabrinnt, und wo es in winzigen, konsolenartigen Bassins kurze Zeit verweilt. Die ganze Kruste ist von üppigen Algenbelägen überzogen. In ihnen wurden festgestellt:

Synecbocystis thermalis Copeland

S. minuscula Woronich

Synecbococcus elongatus Näg.

Chamaesipbon polymorphus Geitl.

Plectonema notatum Schmidle

Pbormidium ambiguum Gom.

Pb. Corium (Ag.) Gom.

$P b$. valderianum (Delp.) Gom.

$P b$. molle Gom.

$P b$. luridum Gom.

$P b$. fragile Gom.

$P b$. tenue (Menegh.) Gom.

$P b$. angustissimum W. et G. S. West

$P b$. bigranulatum Gardner (?)

(gleiche Form wie im Kurbrunnen)

Oscillatoria Willei Gardner em. Drouet

o. ampbibia Ag. ex Gom.

o. geminata Menegh. ex. Gom.

O. terebriformis Ag. ex Gom.

o. formosa Bory ex Gom.

O. animalis Ag. ex Gom.

$O$. pseudoangusta Claus

Pseudanabaena galeata Böcher

P. loncboides Anagnostidis

Beggiatoa minima Winogr.

B. leptomitiformis (Menegh.) Trevis

B. nivea (Rabenh.) Winogr.

Thiotbrix tenuis Winogr.

Spirogyra sp.

\section{Leukerbad}

Die vier ergiebigsten warmen Quellen von Leukerbad $[2-4,6]$ fördern täglich zusammen etwa 20000001 Wasser zutage. Mehr als die Hälfte davon entfällt auf die St.-Lorenz-Quelle, die mitten im Dorf vor dem 
Hôtel Maison-Blanche entspringt und mit gegen $50^{\circ} \mathrm{C}$ Wassertemperatur die heisseste Quelle der Schweiz darstellt. Einige Angaben über sie finden sich in Tab. 2.

Tabelle 2

Das Thermalmasser der St.-Lorenz-Quelle pon Leukerbad (gekürzt nach GÜBELI und DORTA-SCHAEPPI [2])

\begin{tabular}{lclc}
\hline Mineralgehalt (mg pro Liter) & & \\
\hline Kationen & & Anionen & \\
Natrium & 23,575 & Chlorid & $6, \mathbf{9} 98$ \\
Magnesium & 38,33 & Fluorid & $\mathbf{2 , 4 4}$ \\
Kalzium & $43 \mathrm{I}, 82$ & Sulfat & II62,9 \\
Strontium & $\mathrm{II}, 04$ & Hydrokarbonat & 96,00 \\
Ubrige Kationen & 4,073 & Ubrige Anionen & 0,398 \\
Summe der Kationen & 508,838 & Summe der Anionen & $\mathbf{1 2 6 7 , 9 3 6}$ \\
Kieselsäure $\mathrm{H}_{2} \mathrm{SiO}_{3}$ & 29,59 & & \\
Total Mineralien & $\mathbf{1 8 0 6 , 3 6 4}$ & & \\
\hline
\end{tabular}

Gase: Stickstoff und wenig Kohlensäure

Reaktion: $\mathrm{pH}=7$, or bei $20^{\circ} \mathrm{C}$

Temperatur: $48,0^{\circ} \mathrm{C}$ (hyperthermal)

\section{Algen aus der Brunnenstube der St.-Lorenz-Quelle}

Die St.-Lorenz-Quelle tritt in einer engen, gemauerten Brunnenstube an die Erdoberfläche. Ein kleines Fenster lässt nur spärliches Licht ins Innere des Raumes treten, dessen dampferfüllte Atmosphäre eine Temperatur von rund $40^{\circ} \mathrm{C}$ aufweist. Die Innenwände sind völlig nass und trotz des geringen Lichtgenusses von einer fast geschlossenen Algentapete überzogen. An der Decke hängen von Kondenswasser völlig durchnässte Algenzapfen von einigen Millimetern bis über I cm Länge gleich winzigen Stalaktiten herab. Die Analyse solcher Zäpfchen ergab folgendes Ergebnis:

Chamaesiphon polymorphus Geitl.

Plectonema notatum Schmidle

Phormidium angustissimum W. et G.S. West

Plectonema notatum, das schon in Baden aufgefallen war, dominierte bei weitem, ja manche «Zapfen» erschienen sozusagen als Reinbestände der Art.

Der blaugrüne Úberzug der Innenwände setzte sich zusammen aus Synecbocystis thermalis Copeland Aphanocapsa fusco-lutea Hansg. Apbanothece nidulans P. Richt.

A. microspora (Menegh.) Rabenh. Pleurocapsa minor Hansg. em. Geitl. Chamaesipbon polymorpbus Geitl. 
Sipbononema polonicum Geitl. Plectonema notatum Schmidle $P$. nostocorum Bornet ex Gom.
Phormidium angustissimum

W. et G.S. West

Symploca tbermalis (Kütz.) Gom.?

An verschiedenen Stellen war der blaugrüne Wandbelag von dunkleren Flecken durchsetzt. In ihnen wurden festgestellt:

Apbanotbece nidulans P. Richt. A. microspora (Menegh.) Rabenh. Gloeocapsa punctata Näg.?

G. gelatinosa Kütz.?

Pleurocapsa minor Hansg. em. Geitl.
Chamaesipbon polymorphus Geitl. plectonema notatum Schmidle

P. nostocorum Bornet ex Gom. phormidium angustissimum

W. et G.S. West

\section{Algen aus dem « Fussbad»}

Am unteren Dorfrand von Leukerbad speist die «Fussbadquelle» [2] eine der Gemeinde gehörende Freiluftanlage, die zwei ausbetonierte Warmwasserbecken umfasst, die hauptsächlich für Fussbäder, aber in beschränktem Ausmasse auch für Freiluftvollbäder benützt werden. Die Wassertemperatur betrug am Tag der Probenentnahme (28. 8. 63) $38^{\circ} \mathrm{C}$.

Die Innenwände der Becken tragen Überzüge von Algen, die an manchen Stellen hautartig dünn, an anderen dickkrustig und mit kleinen Zäpfchen versehen erscheinen.

InProben derdünnhäutigen Überzüge wurden folgendeFormen bestimmt:

Synecbocystis aquatilis Sauv.

S. minuscula Woron.

Gloeocapsa thermalis Lemmermann

Cbroococcus minor (Kütz.) Näg.

Cb. minutus (Kütz.) Näg.

Chamaesiphon polymorpbus Geitl.

Calotbrix sp.

Plectonema notatum Schmidle

P. nostocorum Bornet ex Gom.

Pbormidium Corium (Ag.) Gom.

$P h$. valderianum (Delp.) Gom.
$P h$. angustissimum W. et G.S. West

$P b$. tenue (Menegh.) Gom.

$P h$. ambiguum Gom.

Pb. molle Gom.

Oscillatoria tenuis Ag. ex Gom.

o. Willei Gardner em. Drouet

Lyngbya martensiana Menegh.

Pseudanabaena catenata Lauterb.

P. galeata Böcher

$P$. lonchoides Anagnostidis

$P$. biceps Böcher

Dickkrustige, mit kleinen Zäpfchen versehene Materialien enthielten

Synecbocystis thermalis Copeland

S. aquatilis Sauv.

S. minuscula Woron.

$S$. crassa Woron.

Synechococcus elongatus Näg.

S. bigranulatus Skuja (?)

Cbroococcus minutus (Kütz.) Näg.

Calotbrix sp.

Plectonema notatum Schmidle
P. nostocorum Bornet ex Gom.

Phormidium tenue (Menegh.) Gom.

$P h$. angustissimum W. et G.S. West

Ph. ambiguum Gom.

Ph. Corium (Ag.) Gom.

Symploca thermalis (Kütz.) Gom.

Pseudanabaena galeata Böcher

$P$. loncboides Anagnostidis

Oscillatoria princeps Vauch. 


\section{Diskussion}

Wie eingangs erwähnt, erheben die vorliegenden Artenlisten keinen Anspruch auf Vollständigkeit: sie basieren auf der Untersuchung von relativ wenigen Proben, und die Frage, ob die aufgefundene Artengarnitur bei eingehender Durchsicht eines reicheren Sammlungsmaterials sich wesentlich ausweiten und in ihrem Aspekt ändern wird, muss offenbleiben. Immerhin ist das starke Überwiegen von Vertretern der Oszillatoriazeen in unseren Listen - 25 von 45 aufgeführten Taxa reihen sich in diese Familie ein - kaum zufällig: So gehören $67 \%$ aller von ANAGNOSTIDIs [I] in griechischen Thermen gefundenen Zyanophytenformen zu den Oszillatoriazeen. Auffallend ist das völlige Fehlen der Gattung Spirulina in unseren Listen, die in griechischen Thermen häufig gefunden wurde. Umgekehrt überrascht die reichliche Entwicklung von Plectonema notatum in beiden untersuchten schweizerischen Thermen, während die Gattung in den griechischen Thermen überhaupt nicht entdeckt wurde.

Vouk [7] versucht, die Thermen biologisch, d. h. auf Grund der in ihnen vorkommenden Lebensgemeinschaften, zu gruppieren, wobei er gleichzeitig auf den individuellen Charakter jeder einzelnen Therme und die damit verbundene Schwierigkeit einer Klassifizierung verweist.

Die Thermen von Baden und Leukerbad sind vorläufig nicht leicht ins System Vouks einzureihen, vor allem weil die natürlichen Wasseraustritte künstlich in dunklen Schächten gefasst sind. Die gesamte lichtbedürftige Vegetation ist daher dort verschwunden.

In Baden konnten sich die Algen nur in den wenigen, ebenfalls künstlich angelegten Kurbrunnen (die zudem von Zeit zu Zeit gereinigt werden) sowie in den auch von Vouk ([7], S. I9) erwähnten Ausflüssen der Thermalquellen am Limmatquai erhalten. Die ursprüngliche Vegetation ist also gewiss nur noch in Relikten vorhanden. Auf Grund der hier vorgelegten Artenlisten wären die Quellen von Baden den Schwefel-Blauthermen (Thio-Zyano- oder SC-Thermen) Vouks beizuordnen.

In Leukerbad fehlen die Schwefelbakterien anscheinend ganz; es liegt offensichtlich eine Blautherme (Cyanophyten- oder C-Therme) vor. Vouk unterscheidet dabei drei Typen, den Mastigocladus-, den Phormidium- und den Oscillatoriatypus. Leukerbad lässt sich auf Grund der festgestellten Arten nicht befriedigend unter diese Typen einreihen. In eingehenderen Untersuchungen wird zu klären sein, ob das auffallende Hervortreten von Plectonema notatum die Aufstellung eines neuen Typus verlangt. 


\section{LITERATURVERZEICHNIS}

[I] ANAGNostidis, K., Untersucbungen über die Cyanopbyceen einiger Thermen in Griecben-

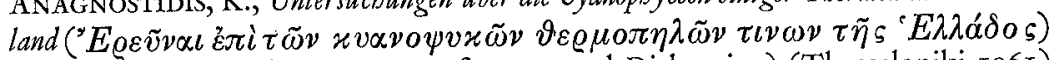
(Griechisch, deutsche Zusammenfassung und Diskussion) (Thessaloniki I96I) p. 322.

[2] GÜBELI, O. und DoRTA-SChaEPPI, Y., Die Neuanalyse scbmeizerischer Mineralquellen, I. Serie, Mitt. aus d. Gebiete der Lebensmittelunters. und Hygiene 41 (H. 3, 4), I-67 (I950).

[3] Die Kurorte der Scbmeiz. Schpoizeriscbes Bäderbuch. Herausgegeben im Auftrag der Schweiz. Ges. Baln. Klimat., unter dem Patronat der Schweiz. Verkehrszentrale, Zürich, 4. Aufl. (Zürich 1930), p. 222.

[4] Die Mineral- und Heilquellen der Scbmeiz. Herausgegeben vom Schweiz. Verein analyt. Chemiker, dem Eidg. Gesundheitsamt und der Schweiz. Ges. Baln. Klimat. (Bern 1937), p. 20 I.

[5] MÜNZEL, U., Die Tbermen von Baden, Eine balneologische Monographie (Selbstverlag, Baden, Schweiz I947), p. 299.

[6] Rivaz, P. DE, und EBENER, A., Leuk-Leukerbad, Sammlung Land und Volk der Schweiz (Neuchâtel o. J.), p. 22.

[7] Vouk, V., Grundriss zu einer Balneobiologie der Thermen (Basel I950), p. 88.

[8] ZEHNDER, A., Drittes Symposium über Fragen der Cyanophytensystematik in Kastanienbaum, x963, Schweiz. Z. Hydrol. 26 (I) I47-I64 (1964).

[9] Desikachary, T. V., Cyanopbyta (New Delhi i959), p. 686.

[IO] ElENkIn, A.A., Monograpbia algarum Cyanopbycearum aquidulcium et terrestrium in finibus U.R.S.S. inventarum. Pars specialis, Fasc. II, 985-I908 (Moskau 1949).

[II] GeITLER, L., Cyanopbyceae, in: Rabenhorsts Kryptogamenflora, 14 (Leipzig I932) p. 1 I96. 